\title{
Desderivación urinaria; de Bricker a Studer. A propósito de dos casos
}

\author{
Cabello Benavente $\mathrm{R}^{*}$, Castillo O**, Pinto I**, Hoyos J**, Vitagliano G**, Díaz $\mathrm{M}^{* *}$, \\ Hernández Fernández $\mathrm{C}^{*}$. \\ *Servicio de Urología. Hospital Gregorio Marañón. Madrid. \\ **Servicio de Urología. Clinica Santa María. Santiago de Chile. Chile.
}

Actas Urol Esp. 2006;30(9):939-942

\section{RESUMEN}

DESDERIVACIÓN URINARIA; DE BRICKER A STUDER. A PROPÓSITO DE DOS CASOS.

Exponemos dos casos de reconversión de una derivación urinaria con asa ileal (Bricker) a neovejiga ortotópica. Exponemos dos casos de reconversión de una derivación urinaria con asa ileal (Bricker) a neovejiga ortotópica, uno mediante cirugía abierta y otro por laparoscopia. En ambos casos el postoperatorio transcurrió sin incidencias. Los dos pacientes se encuentran satisfechos con su nueva situación, continentes y sin presentar complicaciones metabólicas.

La reconversión de un conducto ileal hacia una neovejiga ortotópica es técnicamente viable tanto por cirugía abierta como por laparoscopia, planteándose como una alternativa válida en pacientes que presentan complicaciones secundarias a la derivación inicial o mala adaptación a la misma.

Palabras clave: Derivación urinaria. Cistectomía. Reservorio urinario continente. Cáncer vesical.

\section{ABSTRACT}

URINARY UNDIVERSION; BRICKER TO STUDER. WITH REGARD OF TWO CASES.

We present two cases of urinary undiversion from an ileal loop (Bricker) to an orthotopic neobladder. Due to miss adaptation to the stoma, two patients demanded reconversion to a continent urinary diversion. We proceed to change their urinary diversion to an ileal neobladder (Studer), one by open surgery and the other by laparoscopic surgery. In both cases immediate postoperative went uneventful. Both patients are continent, satisfied with their new situation, and without metabolic complications.

Urinary undiversion from an ileal conduit to an orthotopic neobladder is technically feasible by open or laparoscopic surgery. It is a valid alternative for patients with complications due to their urinary diversion or miss adaptation to the cutaneous stoma.

Keywords: Urinary diversion. Cystectomy. Continent urinary reservoir. Bladder cancer.

$\mathrm{E}$ conducto ileal ha sido durante muchos años la derivación urinaria de elección tras la cistectomía radical ${ }^{1}$.

La reconstrucción vesical ortotópica ha ido aumentando su popularidad, hasta que en la actualidad es considerada como el método de elección para la derivación de pacientes seleccionados $^{2}$, a pesar de su alta complejidad quirúrgica ${ }^{3}$.
La aparición de complicaciones en la derivación urinaria o la mala imagen corporal obliga a considerar, la posibilidad de reconversión de la derivación heterotópica incontinente tipo Bricker, hacia diversos métodos de derivación continente tanto heterotópicos ${ }^{4-8}$ como ortotópicos ${ }^{9-11}$. Presentamos dos pacientes que fueron sometidos a una cirugía de reconversión hacia una neovejiga ileal tipo Studer. 


\section{CASO CLÍNICO}

Caso 1

Varón de 57 años. Con antecedentes de nefroureterectomía derecha por carcinoma de células transicionales en 1991 y cistoprostatectomía radical con derivación urinaria tipo Bricker en 1995 (pTis No Mo, realizada por presentar un carcinoma in situ vesical refractario a BCG). Acudió a nuestra consulta para valorar reconvertir su derivación urinaria, al presentar prolapso del estoma y referir que su vida estaba muy limitada por el dispositivo autoadhesivo externo.

Se solicitó un TAC toraco-abdominal como estudio de re-estadificación, no encontrando signos de actividad tumoral. Su creatinina basal era de $1,5 \mathrm{mg} / \mathrm{dl}$. Se realizó una laparoscopia exploradora en Mayo 2003, con disección completa de la pelvis, logrando identificar el muñón uretral con un Benique. Resecamos el muñón uretral y mediante biopsia intraoperatoria se descartó que presentara infiltración tumoral. La neovejiga (Studer) se construyó tras exteriorizar $45 \mathrm{~cm}$ de íleon terminal por el orificio del estoma. La anastomosis uretra-neovejiga se realizó de forma laparoscópica. El tiempo quirúrgico total fue de 480 minutos, con un sangrado estimado de 100 cc (Figs. 1 y 2). El paciente fue dado de alta al sexto día postoperatorio sin complicaciones.

La evolución desde entonces ( 29 meses) ha sido favorable, sin evidenciar alteraciones metabólicas ni signos de recidiva de la enfermedad basal.

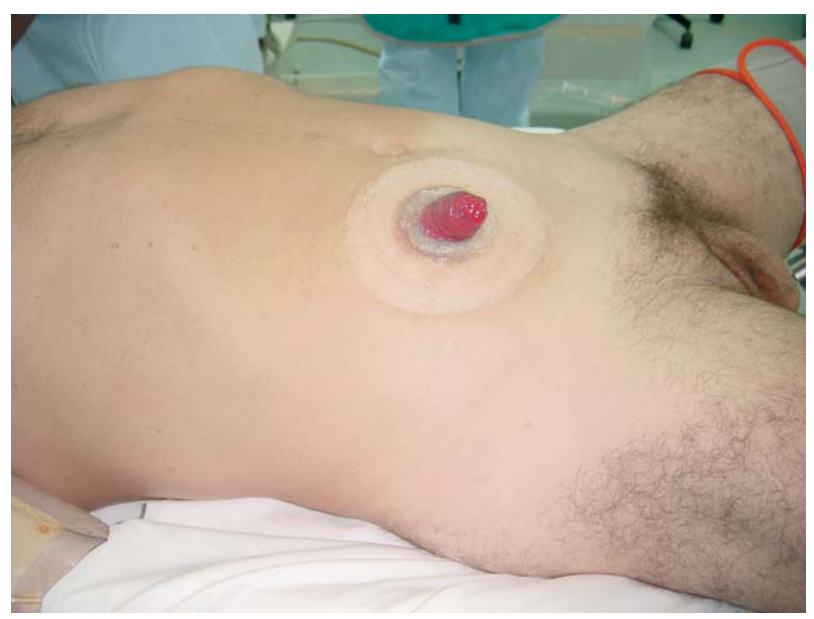

FIGURA 1: Aspecto preoperatorio del paciente 1 en la mesa quirúrgica.

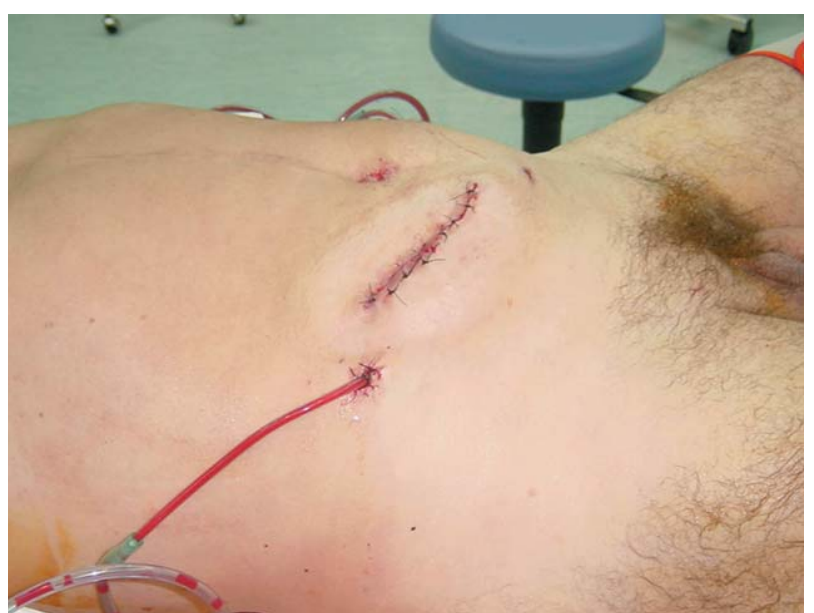

FIGURA 2: Paciente 1 tras la desderivación. Cierre del defecto de la pared abdominal donde se localizaba el estoma. Exteriorización de tubo de drenaje por orificio del trocar colocado en la fosa iliaca derecha.

\section{Caso 2}

Varón de 63 años. Consultó por hematuria macroscópica monosintomática. Se solicitó una ecografía donde se apreció una masa de $5 \mathrm{~cm}$ en cara lateral izquierda de la vejiga que producía una ureterohidronefrosis en el riñón izquierdo. Realizamos una RTU-biopsia, objetivando un tumor vesical infiltrante de alto grado. El estudio de extensión resultó negativo. La creatinina del paciente era de $2,5 \mathrm{mg} / \mathrm{dl}$. Se realizó una cistoprostatectomía radical laparoscópica con derivación urinaria tipo Bricker en marzo 2005 (pT2b No Mo de alto grado, afectando a zona trigonal y cara posterior).

El curso operatorio evolucionó favorablemente. En la revisión postoperatoria al mes de la cirugía, se redujo la ureterohidronefrosis izquierda manteniendo cierto grado de atrofia cortical, y mejoró la función renal (creatinina de 1,65 mg/dl) (Figs. 3 y 4).

$\mathrm{El}$ paciente presentaba muy mala tolerancia al estoma, insistiendo en su conversión en una derivación continente. Situación que nos planteamos por la mejoría de su función renal. A los dos meses realizamos una laparotomía exploradora, logrando una buena disección pélvica e identificación del muñón uretral. Construimos una neovejiga ileal con $45 \mathrm{~cm}$ de íleon. El tiempo quirúrgico fue de 180 minutos, con un sangrado menor de $80 \mathrm{cc}$. El paciente fue dado de alta al sexto día postoperatorio sin presentar complicaciones. 


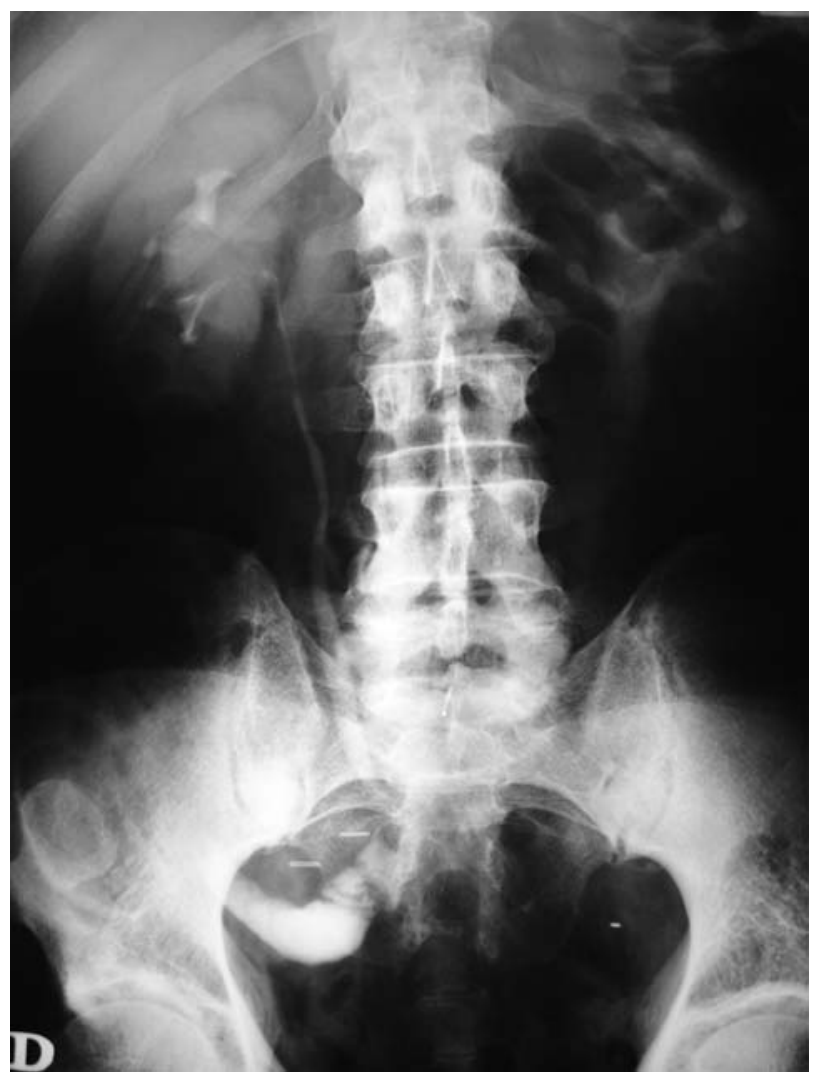

FIGURA 3: Urografia intravenosa del paciente 2 con la derivación urinaria tipo Bricker.

A los 5 meses de la cirugía, está continente durante el día y la noche, con buen vaciamiento del reservorio.

Ambos pacientes fueron concienzudamente informados de los riesgos de la cirugía y de sus posibles complicaciones, así como de las potenciales secuelas en términos de trastornos metabólicos, diarrea persistente e incontinencia y/o necesidad de autocateterismos por alto residuo post-miccional. En ambos, el asa de Bricker se liberó de la pared abdominal y tras acortarlo se anastomosó a la chimenea de la neovejiga, no precisando de reimplante ureteral. La sonda vesical se retiró a las 3 semanas, logrando ambos la continencia en corto espacio de tiempo. Los dos pacientes están muy satisfechos con la derivación ortotópica.

\section{DISCUSIÓN}

En la consulta de urología vemos pacientes con derivaciones urinarias no continentes, sin signos de recidiva de su tumor vesical que por diversos

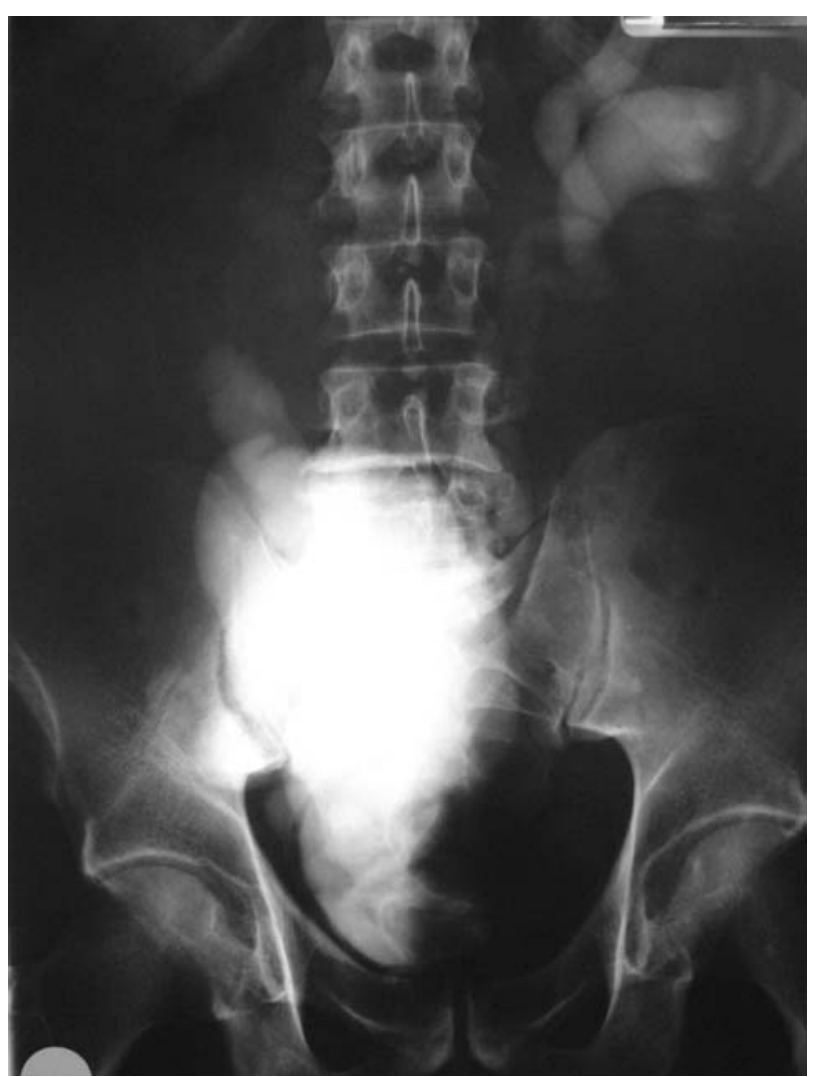

FIGURA 4: Urografia intravenosa de control en fase tardía del paciente 2 tras la desderivación hacia neovejiga ileal continente.

motivos precisan o solicitan una derivación continente. En estos individuos se han comunicado desderivaciones tanto hacia dispositivos heterotópicos continentes $^{4-8}$ como ortotópicos $^{9-11}$. La elección del tipo de dispositivo y la vía de abordaje debe realizarse a criterio del cirujano y de las preferencias del paciente. La vía laparoscópica presenta inconvenientes como un tiempo operatorio mayor y que puede resultar técnicamente más compleja, aunque facilitará la exactitud de la disección pélvica y de la anastomosis uretro-neovejiga.

El motivo de la desderivación, debe estar justificado, siempre en un paciente oncologicamente controlado, con bajo riesgo de recidiva uretral y que se considere candidato en términos de edad, situación social y estado general de salud ${ }^{4}$. Entre las causas que pueden hacer plantear la desderivación a un enfermo se encuentran las complicaciones derivadas del estoma (estenosis estomal, dermitis paraestomal, hernia paraestomal, mala tolerancia a los dispositivos autoadhesivos), de la anastomosis urétero-ileal (estenosis, litiasis, reflujo), o 
por problemas derivados de la baja autoestima y el deterioro en la percepción de la imagen corporal segundaria al estoma. En una reciente revisión Poter et al. ${ }^{12}$ no han encontrado evidencia suficiente en la literatura para concluir que un método de derivación urinaria es superior al resto en términos de calidad de vida. No obstante, previamente se había sugerido que los pacientes a los que se practicaba una derivación continente tipo Studer tenían diferencias estadísticamente significativas a su favor, respecto a los que se derivaban mediante Bricker en términos emocionales y de interacciones/relaciones sociales ${ }^{13}$.

Estos pacientes presentan un mayor riesgo de presentar complicaciones por la necesidad de utilizar amplios segmentos intestinales, al sumarse el utilizado para el Bricker y el Studer. Tales complicaciones, como el síndrome de mal absorción o la diarrea secundaria a intestino corto, tienen repercusiones clínicas (litiasis biliar y renal) y metabólicas (déficit de vitamina B12, de folato...). No obstante son complicaciones raras, incluso en series que utilizan para la desderivación segmentos intestinales que incluyen la válvula íleo-cecal ${ }^{5-8}$. Para minimizar este riesgo se ha descrito la utilización de parte del asa de Bricker, anastomosándolo a la nueva bolsa continente ${ }^{6-8}$, técnica que utilizamos en los dos pacientes. En nuestra opinión, este hecho además de ahorrar intestino, facilita la cirugía y reduce la posibilidad de complicaciones al no precisar un nuevo reimplante ureteral. Otras situaciones no resultan tan excepcionales, como la hipercloremia moderada o la acidosis metabólica ${ }^{5}$. No obstante, no debe animarse a los pacientes con un conducto ileal normofuncionante a someterse a esta cirugía, puesto que se trata de una cirugía mayor no exenta de complicaciones ${ }^{5}$. En el estudio preoperatorio previo se debe asegurar la ausencia de recidiva uretral así como la integridad de la misma ${ }^{11}$. El tiempo transcurrido entre la cistectomía y la desderivación, no parece afectar negativamente a sus resultados ${ }^{10}$.

En la mayoría de los casos reportados se afirma que la desderivación aumentó significativamente la calidad de vida ${ }^{5}$. Uno de los principales factores a este respecto es la consecución del posterior control voluntario de la micción. La continencia se ha puesto en relación con una adecuada y meticulosa anastomosis uretro-intestinal, así como con la presencia del ápex residual prostático que facilite la misma ${ }^{10}$. Dos instrumentos útiles para tranquilizar tanto al cirujano como al paciente es la posibilidad de colocar un esfinter artificial (en caso de incontinencia por lesión severa del aparato esfinteriano en la disección uretral pélvica) o de realizar autocateterismos (en caso de incontinencia secundaria a mal vaciamiento de la neovejiga) ${ }^{10}$.

\section{CONCLUSIÓN}

La realización de una reconversión de un conducto ileal hacia una neovejiga ortotópica es técnicamente viable, ya sea mediante laparoscopia o cirugía abierta. A pesar de su complejidad técnica se puede considerar como una alternativa válida en pacientes que presentan complicaciones secundarias a la derivación inicial o mala adaptación a la misma.

\section{REFERENCIAS}

1. Bricker EM. Bladder substitution alter pelvis evisceration. Surg Clin North Am. 1950;30:1511-1521.

2. Hautmann RE. Urinary diversion; ileal conduit to neobladder. J Urol. 2003;169(3):834-842.

3. Kulkarni JN, Pramesh CS, Rathi S, Pantvaidya GH. Long-term results of orthotopic neobladder reconstruction after radical cystectomy. BJU Int. 2003;91(6):485-488.

4. Benezra V, Lambrou NC, Salom EM, Penalver MA. Conversion of an incontinent urinary conduit to a continent urinary reservoir (Miami pounch). Gynecol Oncol. 2004;94(3):814-817.

5. Pow-Sang JM, Helal M, Figueroa E, Sanford E, Persky L, Lockhart I Conversion from external appliance wearing or internal urinary diversión to a continent urinary reservoir (Florida pouch I and II): surgical technique, indications and complications. J Urol. 1992;147(2):356-360.

6. Oesterling JE, Gearhart JP. Utilization of ileal conduit in construction of continent urinary reservoir. Urol. 1990;36(1):15-19.

7. Pahernik S, Stein R, Hohenfellner M, Thuroff JW. Conversion from colonic or ileal conduit to continent cutaneous urinary diversion. J Urol. 2004;171(6Pt1):2293-2297.

8. Ahlering TE, Gholdoian G, Skarecky D, Weinberg AC, Wilson TG. Simplified technique with short and long-term follow-up of convertion of an ileal conduit to an Indiana pouch. J Urol. 2000;163:1428-1431.

9. López AE, Gutiérrez E, Flores I. Neovejiga ortotópica en una paciente después de radioterapia y exenteración anterior con conducto ileal. Arch Esp Urol. 1990;14:80-83.

10. Boyd SD, Esrig D, Stein JP. Undiversion in men following prior cystoprostatectomy and cutaneus diversion. Is it practical?. J Urol. 1994; 152:334-337.

11. Rioja Sanz LA, Liédana Torres JM, González Enguita C. Urinary undiversion. Actas Urol Esp. 1990;14:80-83.

12. Porter MP, Penson DF. Heath related quality of life after radical cystectomy and urinary diversión for bladder cancer; a systematic review and critical análisis of the literature. J Urol. 2005;173:1318-1322.

13. Yoneda T, Igawa M, Shiina H, Shigeno K, Urakami S. Postoperative morbidity, funcional results an quality of life of patients following orthotopic neobladder reconstruction. Int J Urol. 2003;10:119-125.

Dr. R. Cabello Benavente

Av. Galaxia 6, esc. 2, $2^{\circ} \mathrm{b}$

28023. Madrid

E-mail: ramirocabello@telefonica.net

(Trabajo recibido el 29 de noviembre de 2005) 